\title{
THE RESIDUAL FINITENESS OF A CLASS OF 1-RELATOR GROUPS
}

\author{
by R. B. J. T. ALLENBY and C. Y. TANG $\dagger$
}

(Received 30 May, 1986)

1. Introduction. In [3] Gilbert Baumslag asserted that, for non-zero $\ddagger$ integers $\alpha, \beta, \gamma, \delta$ such that $\alpha+\gamma \neq 0 \neq \beta+\delta$, the group $G=\left\langle a, b: a^{\alpha} b^{\beta} a^{\gamma} b^{\delta}\right\rangle$ is residually finite (RF). This result has been quoted in the literature: for example, in [2]. At the "Groups '85" meeting at St. Andrews, the second author learned, indirectly, that Professor Baumslag could not recall all the details of the rather complicated (unpublished) proof he had constructed and that he referred those asking for a proof to the present authors. It thus seems worthwhile formally to record the following fairly short proof of the above claim.

2. The proof. We first observe that by forming $G_{1}=\langle x:\rangle *_{x^{\beta+\delta}=a} G$ and $G_{2}=$ $G_{1} *_{b=y a+\gamma}\langle y:\rangle$, we embed $G$ into the group $E=\left\langle x, y: x^{A} y^{B} x^{C} y^{D}\right\rangle$ where $A+C=$ $B+D=(\alpha+\gamma)(\beta+\delta) \neq 0$. It then suffices to prove $E$ is RF. Without loss of generality we may suppose that $A+C=B+D>0$, that $A \geqslant B>0$ and that $A \geqslant B \geqslant D \geqslant C$. If we set $y=x^{-1} s$ we have $E=\left\langle x, s: x^{A}\left(x^{-1} s\right)^{B} x^{C}\left(x^{-1} s\right)^{D}\right\rangle$. Since the total exponent sum on $x$ in the relator is zero, we may regard $E$ as an HNN extension, by $\langle x\rangle$, of a certain base group $\mathfrak{b}$.

Suppose, for the moment, that $A>B$ so that $D>C$. Writing $s_{i}=x^{i} s x^{-i}$, as usual, we find that:

(1) If $D>0$ then $A-B+C-1 \geqslant 0$ and

$$
\mathfrak{b}=\left\langle s_{A-1}, s_{A-2}, \ldots, s_{0}: s_{A-1} \ldots s_{A-B} \cdot s_{A-B+C-1} s_{A-B+C-2} \ldots s_{0}\right\rangle .
$$

Here the associated (free) subgroups are $H=\left\langle s_{A-1}, \ldots, s_{1}\right\rangle$ and $K=\left\langle s_{A-2}, \ldots, s_{0}\right\rangle$.

(2) If $D<0$ (so that $C<0$ ) then $B>C+1$ and

$$
\mathfrak{b}=\left\langle s_{A-1}, \ldots, s_{A-B+C}: s_{A-1} \ldots s_{A-B} s_{A-B+C}^{-1} s_{A-B+C+1}^{-1} \ldots s_{-1}^{-1}\right\rangle \text {, }
$$

with associated subgroups $H=\left\langle s_{A-1}, \ldots, s_{A-B+C+1}\right\rangle$ and $K=\left\langle s_{A-2}, \ldots, s_{A-B+C}\right\rangle$.

Note that, in each of these cases, the presence in the relator of a single occurrence of both the generator with the maximum suffix and that with the minimum suffix imply that $H=K$. Thus $H=K=\mathfrak{b}$ and $H$ is normal in $G$. Hence $G$ is an infinite cyclic extension of a finitely generated free group and so is well known to be RF.

Thus we may suppose that $A=B$ and $C=D$. If $A=B=C=D$, we have the residual finiteness of $G$ by Corollary 3 of [1]. Otherwise we have $A=B>C=D$ so that $E$ is an

$\dagger$ The work of the second author was partly supported by Grant No. A-4064 from the NSERC of Canada. $\ddagger$ If any one of $\alpha, \beta, \gamma, \delta$, is zero $G$ reduces to a group well known to be residually finite.

Glasgow Math. J. 29 (1987) 267-269. 
HNN extension by $\langle x\rangle$ of the base group

$$
\begin{aligned}
& \mathfrak{b}_{>0}=\left\langle s_{A-1}, \ldots, s_{0}: s_{A-1} s_{A-2} \ldots s_{0} \cdot s_{C-1} \ldots s_{0}\right\rangle \text { if } C=D>0 \text { or } \\
& \mathfrak{b}_{<0}=\left\langle s_{A-1}, \ldots, s_{0}, \ldots, s_{C}: s_{A-1} \ldots s_{0} \cdot s_{C}^{-1} \ldots s_{-1}^{-1}\right\rangle \text { if } C=D<0 .
\end{aligned}
$$

In the latter case, we again have a single occurrence of the maximum and minimum suffices, showing that $\mathfrak{b}_{<0}$ is RF.

Now look at $\mathfrak{b}_{>0}$. The associated subgroups are $H=\left\langle s_{A-1}, \ldots, s_{1}\right\rangle$ and $K=$ $\left\langle s_{A-2}, \ldots, s_{0}\right\rangle$ and $H$ and $K$ are free on these generators. If we set, for $0 \leqslant i \leqslant A-1$, $u_{i}=s_{i} s_{i-1} \ldots s_{0}$ we find that $K$ is freely generated by $\left\{u_{A-2}, \ldots, u_{0}\right\}$ and $H$ is freely generated by

$$
\left\{u_{A-1} u_{0}^{-1}, u_{A-2} u_{0}^{-1}, \ldots, u_{C-1} u_{0}^{-1}, \ldots, u_{1} u_{0}^{-1}\right\}
$$

hence by

$$
\left\{u_{C-1}^{-1} u_{0}^{-1}, u_{A-2} u_{0}^{-1}, \ldots, u_{C-1} u_{0}^{-1}, \ldots, u_{1} u_{0}^{-1}\right\}
$$

since the relation in $\mathfrak{b}_{>0}$ is $u_{A-1} u_{C-1}=1$. Thus $H$ is freely generated by

$$
\begin{aligned}
\left\{\left(u_{C-1}^{-1} u_{0}^{-1}\right)\left(u_{C-1} u_{0}^{-1}\right)^{-1}, u_{A-2} u_{0}^{-1}, \ldots, u_{C-1} u_{0}^{-1}, \ldots, u_{1} u_{0}^{-1}\right\} & \\
& =\left\{u_{C-1}^{2}, u_{A-2} u_{0}^{-1}, \ldots, u_{1} u_{0}^{-1}\right\} .
\end{aligned}
$$

Note that, since $s_{A-1}=u_{C-1}^{-1} u_{A-2}^{-1}$, we have $H \leqslant K=x^{-1} H x$. Thus the normal closure $H^{G}$ of $H$ in $G$ is the ascending union of isomorphic finitely generated free groups, $\bigcup_{i=0}^{\infty} x^{-i} H x^{i}$.

Now suppose $g(\neq 1)$ is an element of $G$. If $g \notin H^{G}$ it is trivial to find a finite homomorphic image, $G \varphi$, of $G$ in which $g \varphi \neq 1$. Thus suppose $g \in H^{G}$. We assume, without loss of generality, that $g \in K$. Since $K$ is finitely generated and free, there exists a verbal subgroup $V(K)=V$, say, of finite odd index in $K$ such that $g \notin V$. Consider $H V / V$. It is generated by

$$
\begin{aligned}
\left\{u_{C-1}^{2} V, u_{A-2} u_{0}^{-1} V, \ldots, u_{C-1} u_{0}^{-1} V,\right. & \left.\ldots, u_{1} u_{0}^{-1} V\right\} \\
& =\left\{u_{C-1} V, u_{A-2} u_{0}^{-1} V, \ldots, u_{C-1} u_{0}^{-1} V, \ldots, u_{1} u_{0}^{-1} V\right\}
\end{aligned}
$$

since $u_{C-1} V$ has odd order. Thus $H V / V$ can be freely generated also by

$$
\left\{u_{C-1} V, u_{A-2} V, \ldots, u_{0}^{-1} V, \ldots, u_{1} V\right\} .
$$

This implies that $H V / V \simeq K / V$. Thus $H /(H \cap V(K)) \simeq K / V(K) \simeq H / V(H)$. Now clearly $V(H) \leqslant H \cap V(K)$. Since $|H /(H \cap V(K))|=|H / V(H)|<\infty \quad$ we deduce that $V(H)=H \cap V(K)$.

Let $W=\bigcup_{i=0}^{\infty} V\left(x^{-i} H x^{i}\right)=V\left(\bigcup_{i=0}^{\infty} x^{-i} H x^{i}\right)=V\left(H^{G}\right)$. Then $W$ is fully invariant in $H^{G}$ and hence normal in $G$. Further, $W$ is such that $\left|H^{G} / W\right|=|H / V(H)|$ as is easily seen. Thus $g W$ is a non trivial element in the group $G / W$ which is an infinite cyclic extension of a finite group and this is RF. This completes the proof. 


\section{REFERENCES}

1. R. B. J. T. Allenby, L. E. Moser and C. Y. Tang, The residual finiteness of certain 1-relator groups, Proc. Amer. Math. Soc. 78 (1980), 8-10.

2. R. B. J. T. Allenby and C. Y. Tang, Residual finiteness of certain 1-relator groups; extensions of results of Gilbert Baumslag, Math. Proc. Cambridge Philos. Soc. 97 (1985), 225-230.

3. Gilbert Baumslag, Some problems on 1-relator groups, Proceedings of the Second International Conference on the Theory of Groups (Lecture Notes in Mathematics 372, Springer, 1974), 75-81.

SChOOL of Mathematics

THE UNIVERSITY

LEEDS LS2 9JT

ENGLAND
Department of Pure Mathematics The University OF WATERLOO WATERLOO

OnTARIO N2L 3G1

Canada 